\section{Zur Kristallstruktur kristalliner Hydroxyde}

Von Robert Fricke

Laboratorium für anorganische Chemie der Technischen Hochschule Stuttgart

(Z. Naturforschg. 3 a, 62 [1948]; eingegangen am 21. Januar 1948)

Bisher wird vielfach noch die Ansicht vertreten, daß das $\mathrm{OH}^{\prime}$-Ion als ein besonders leicht polarisierbares Ion sich in der Reihe $\mathrm{F}^{\prime}, \mathrm{Cl}^{\prime}, \mathrm{Br}^{\prime}, \mathrm{J}^{\prime}$ etwa dem Jodion an die Seite stellen lasse und daß demzufolge die Hydroxyde alle in Schichtengittern kristallisierten. Tatsächlich kristallisieren ja auch viele Hydroxyde in ausgesprochenen Schichtengittern, wie z. B. Ca $(\mathrm{OH})_{2}$, $\mathrm{Cd}(\mathrm{OH})_{2}, \mathrm{Co}(\mathrm{OH})_{2}, \mathrm{Fe}(\mathrm{OH})_{2}, \mathrm{Mg}(\mathrm{OH})_{2}, \mathrm{Mn}(\mathrm{OH})_{2}$, $\mathrm{Ni}(\mathrm{OH})_{2}$ und $\alpha-\mathrm{Zn}(\mathrm{OH})_{2}$ im $\mathrm{C}_{6}$-Typ des $\mathrm{CdJ}_{2}{ }^{1}$ oder auch der Hydrargillit $\mathrm{Al}(\mathrm{OH})_{3}{ }^{2}$ und das $\mathrm{LiOH}^{3}$.

Doch ist seit längerem bereits die Struktur eines Hydroxydes, und zwar des stabilsten $\mathrm{Zn}(\mathrm{OH})_{2}$ $\left[\varepsilon-\mathrm{Zn}(\mathrm{OH})_{2}\right]$, aufgeklärt, welches absolut nicht als Schichtengitter aufgefaßt werden kann ${ }^{4}$. Hier ist jedes Metallion etwas verzerrt tetraedrisch von $4 \mathrm{OH}^{\prime}$ umgeben, während jedes $\mathrm{OH}^{\prime}$ als nächste Nachbarn 2 Metallionen und $2 \mathrm{OH}^{\prime}$ hat.

Absolut keine Schichtengitter bilden weiter die in jüngster Zeit genügend großkristallin erhaltenen und in ihrer Struktur aufgeklärten Hydroxyde des Y und der Lanthaniden vom Typ $\mathrm{Me}(\mathrm{OH})_{3}{ }^{5}$. Hier ist jedes Metallion in erster Sphäre von $9 \mathrm{OH}^{\prime}$ umgeben, von denen 6 besonders eng benachbart in zwei Gruppen von je 3 ein gleichseitiges Dreieck bildenden $\mathrm{OH}^{\prime}$ über und unter dem Metallion liegen (dabei ein trigonales Prisma bildend), während die drei anderen, etwas entfernteren $\mathrm{OH}^{\prime}$ der ersten Sphäre um das Metallion den gleichen c-Index haben, also auf gleicher "Höhe“ liegen wie das Metallion, und jedes zu einem anderen $\mathrm{OH}^{\prime}$-Dreieck gehört. Die hexagonale Elementarzelle enthält 2 Moleküle $\mathrm{Me}(\mathrm{OH})_{3}$ (vgl. Abb. 1).

Ebenfalls absolut kein Schichtengitter bilden die isotypen Hydroxyde $\mathrm{Sc}(\mathrm{OH})_{3}$ und $\operatorname{In}(\mathrm{OH})_{3}$. Sie kristallisieren regulär mit 8 Molekülen $\mathrm{Me}(\mathrm{OH})_{3}$ in der teilweise raumzentrierten Elementarzelle ${ }^{6}$. Ihre Struktur $^{7}$ ist in Abb. 2 wiedergegeben.

Man erkennt hier, wie je $12 \mathrm{OH}^{\prime}$ als Dreiecke zu Ikosiëdern zusammengefaßt sind, während jedes Metallion oktaëdrisch von $6 \mathrm{OH}^{\prime}$ umgeben ist.

Die Besonderheit der beiden zuletzt angeführten Strukturen, nämlich die Zusammenfassung der $\mathrm{OH}^{\prime}$ $\mathrm{zu}$ geschlossenen Gruppen, kann nur verstanden werden auf Grund der Wasserstoffbindung zwischen den einzelnen O-Ionen. Jeder Wasserstoff ist hier zwei $\mathrm{O}$ gemeinsam ${ }^{8}$.

Hydroxyde bilden also in vielen Fällen kein Schich-

${ }^{1}$ Fricke-Hüttig, Hydroxyde und Oxydhydrate, Akad. Verlags-Gesellsch. Leipzig 1937, insbesondere S. $503 \mathrm{ff}$.

${ }^{2}$ H. D. M e g a w, Z. Kristallogr. 87, 185 [1934].

3 Th. Ernst, Z. physik. Chem., Abt. B, 20, 65 [1933].

${ }^{4}$ H. D. M e g a w, Z. Kristallogr. 90, 283 [1935]; R. B. Core y u. R. W. Wy ck off, Z. Kristallogr. 86, 8 [1933]; K. P es tre cov, Z. Kristallogr. 91, $505[1935]$.

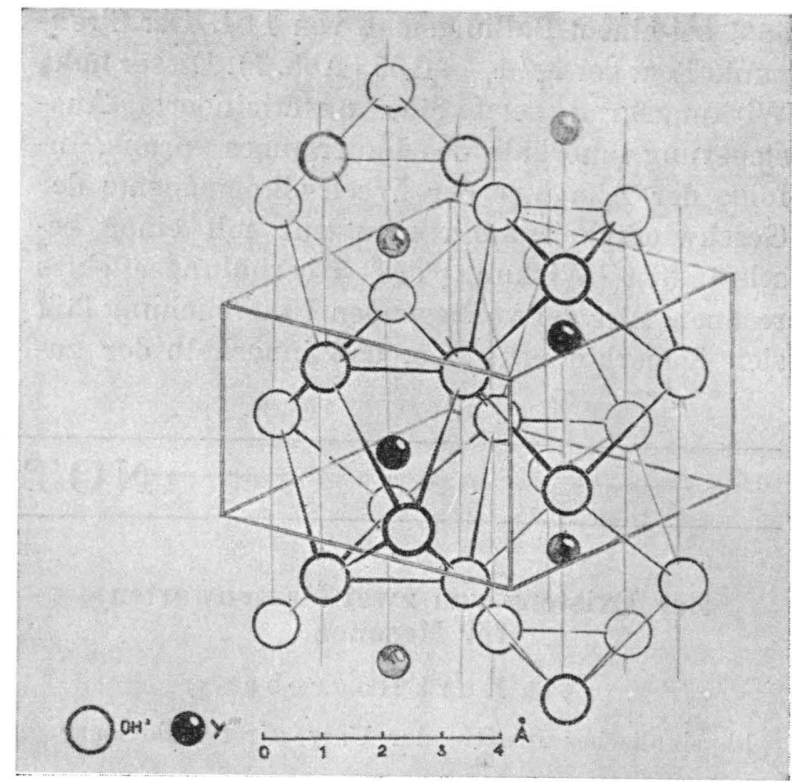

Abb. 1.

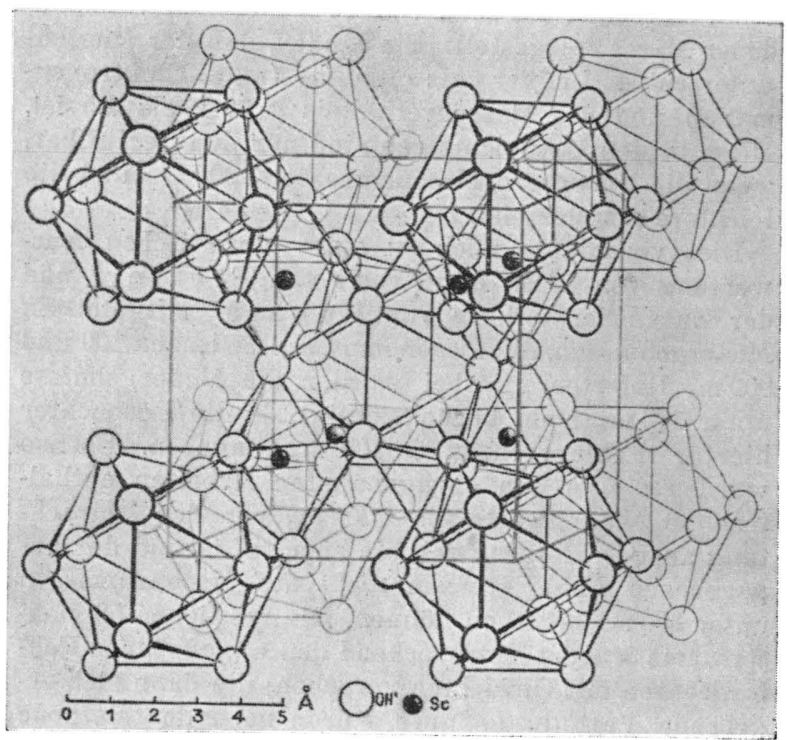

Abb. 2.

tengitter, weil sich das $\mathrm{OH}^{\prime}$ nicht ohne weiteres mit einem einfachen, gut polarisierbaren Ion, wie dem $\mathrm{J}^{\prime}$, vergleichen läßt.

${ }^{5}$ R. Fricke u. A. Se itz, Z. anorg. allg. Chem. 254, 107 [1947]; K. S chubert u. A. Seitz, Z. anorg. allg. Chem. 254, 116 [1947].

${ }_{6}$ R. Fricke u. A. Seitz, Z. anorg. allg. Chem. 255, 13 [1947]; vgl. auch Z. Naturforschg. 2 a, 535 [1947].

7 K Schubert u. A. Seitz, Z. anorg. allg. Chem. 256, Heft 4 [1948].

8 J. D. Bernal u. H. D. Megaw, Proc. Roy. Soc. [London], Ser. A, 151, 384 [1935]]. 